\title{
INFLUENCE DE LA LUMINOSITÉ NOCTURNE ET DE LA TURBIDITÉ SUR LE COMPORTEMENT VERTICAL DE MIGRATION DE LA CIVELLE D'ANGUILLE (ANGUILLA ANGUILLA L.) DANS L'ESTUAIRE DE L'ADOUR.
}

\author{
M.N. DE CASAMAJOR (1), N. BRU (2) et P. PROUZET (1)
}

(1) Laboratoire IFREMER, Station d'Hydrobiologie, B.P. 3, 64310 Saint-Pée-sur-Nivelle, France.

(2) Université de Pau et des Pays de l'Adour, Laboratoire de Mathématiques Appliqués, Avenue de l'Université, 64000 Pau, France.

\section{RÉSUMÉ}

Les mouvements migratoires des civelles en fonction des conditions environnementales sont étudiés à partir d'une campagne d'échantillonnage dans l'estuaire de l'Adour au cours de la saison 1997/1998. Cette campagne se déroule la nuit, pendant le flot, à $10 \mathrm{~km}$ en amont de la limite transversale de la mer, sur un secteur de $12 \mathrm{~km}$, situé dans la zone de propagation de la marée.

Les densités de civelles sont estimées à partir du poids capturé dans des filets à mailles fines par unité de volume d'eau filtré dans différents secteurs ( 5 stations, 3 traits de tamis par station et 2 prélèvements par trait de tamis en surface et en profondeur). Entre novembre et mars, les arrivées de civelles forment une courbe en cloche avec un pic de migration en janvier. Le flux de civelles transite dans cette zone, en surface et en profondeur, mais préférentiellement en profondeur si l'intensité de la lumière nocturne (phase lunaire) est forte et la turbidité de l'eau faible. La situation lunaire et la turbidité modifient le comportement des individus ainsi que leur accessibilité à la pêcherie.

Compte tenu des observations effectuées, le schéma migratoire vertical de l'espèce peut être modélisé de la manière suivante : l'interaction entre la luminosité nocturne et la turbidité influence les déplacements de civelles dans la colonne d'eau en relation avec leur comportement lucifuge. Plus la lumière nocturne qui pénètre dans la colonne d'eau est importante et plus les civelles migrent en profondeur.

Mots-clés : civelle, comportement migratoire, cycle lunaire, densité, estuaire de l'Adour, turbidité. 


\title{
INFLUENCE OF NIGHT BRIGHTNESS AND TURBIDITY ON THE VERTICAL MIGRATORY BEHAVIOUR OF GLASS-EELS (ANGUILLA ANGUILLA L.) IN THE ADOUR ESTUARY.
}

\begin{abstract}
Glass-eels migratory movements according to environmental conditions were studied on Adour estuary during the 1997/1998 catch season. These observations took place at night, during flood tide, 10 kilometres upstream the sea transversal limit, on a sector of 12 kilometres in length where we observed the propagation of tide.
\end{abstract}

Glass-eels densities (in $\mathrm{g}$ per $100 \mathrm{~m}^{3}$ ) were estimated with fine mesh nets on 5 sampling stations. On each station, 3 longitudinal transects were sampled simultaneously in surface and close to the bottom. Catch abundance between November and March followed a dome shape tendency with a pick in January. Movements into the water column were induced by moonlight intensity. Glass-eels migrate mainly close to the bottom when turbidity is low and moonlight intensity is high. Moon phases and turbidity induced modifications in glass-eels behaviour and catchability by the fishery.

According to these observations, a modelisation of vertical migratory scheme of glass-eels in that estuary can be proposed : high moonlight intensity and low turbidity induced abundance of glass-eels mainly close to the bottom ; low moonlight intensity and/or high turbidity induced dispersion of glass-eels throughout the water column. turbidity.

Key-words : glass-eel, migratory behaviour, moon phase, density, Adour estuary,

\section{INTRODUCTION}

Les caractéristiques des civelles en migration sur le bassin de l'Adour ont été étudiées à partir de captures au tamis à main en surface (CHARLON, 1980 ; CHARLON et BLANC, 1982). Les déplacements de civelles dans la colonne d'eau sont analysés à partir de captures par unité d'effort des marins-pêcheurs dans la partie fluviale de l'estuaire (BRU et al., 1996 ; DE CASAMAJOR et al., 1996) et d'une première campagne d'échantillonnage pendant la saison de migration 1996/1997 (BRU et al., 1997 ; DE CASAMAJOR, 1998).

Des travaux antérieurs montrent l'existence d'un comportement lucifuge des civelles lors de leur traversée de l'estuaire (CANTRELLE, 1981 ; GANDOLFI et al., 1984). L'influence du rythme lunaire sur les rythmes d'activité des poissons est un phénomène connu (GIBSON, 1978). L'influence du cycle lunaire sur le comportement migratoire des civelles est souvent étudiée à partir de données de captures (FERNANDEZ et VASQUEZ, 1978 ; JELLYMAN, 1979 ; TZENG, 1985).

La turbidité est peu étudiée. Ce paramètre modifie la pénétration de la lumière dans l'eau en fonction de la nature et la quantité de sédiments en suspension dans le milieu. Notre objectif est de montrer que les modifications de la lumière ambiante, induites par la variabilité de ce facteur, jouent un rôle primordial sur les mouvements verticaux des civelles dans la colonne d'eau. Ces modifications de la pénétration de la lumière résultent de la variabilité de la clarté nocturne dépendante du cycle lunaire et de la nébulosité.

Nous présentons ici les résultats de la campagne de pêche 1997/1998 réalisée en partie basse de l'estuaire. Nous étudions les déplacements des civelles dans la colonne d'eau à partir des proportions de densités de civelles au fond et en surface dans différents secteurs de l'estuaire en fonction des conditions environnementales, et plus 
particulièrement de la turbidité et du cycle lunaire. Une analyse statistique descriptive de ces données précise les relations entre les facteurs environnementaux et le comportement lucifuge des civelles.

\section{MATÉRIEL ET MÉTHODES}

\section{Cadre géographique}

Le bassin de l'Adour est le $5^{\text {eme }}$ réseau hydrographique de France (bassin versant de $\left.16733 \mathrm{~km}^{2}\right)$, il se localise dans le Sud-Ouest de la France $\left(43^{\circ} 30^{\prime} \mathrm{N}\right.$ et $\left.1^{\circ} 32^{\prime} \mathrm{W}\right)$. L'Océan Atlantique et la chaîne des Pyrénées provoquent une augmentation des précipitations du Nord au Sud et d'Ouest en Est. Ainsi, les précipitations permettent à l'Adour de garder un débit moyen soutenu toute l'année (supérieur à $300 \mathrm{~m}^{3} / \mathrm{s}$ ).

L'Adour présente l'avantage d'être un estuaire de taille réduite, ce qui facilite la prospection et limite les biais introduits par un échantillonnage peu représentatif du milieu (Figure 1).

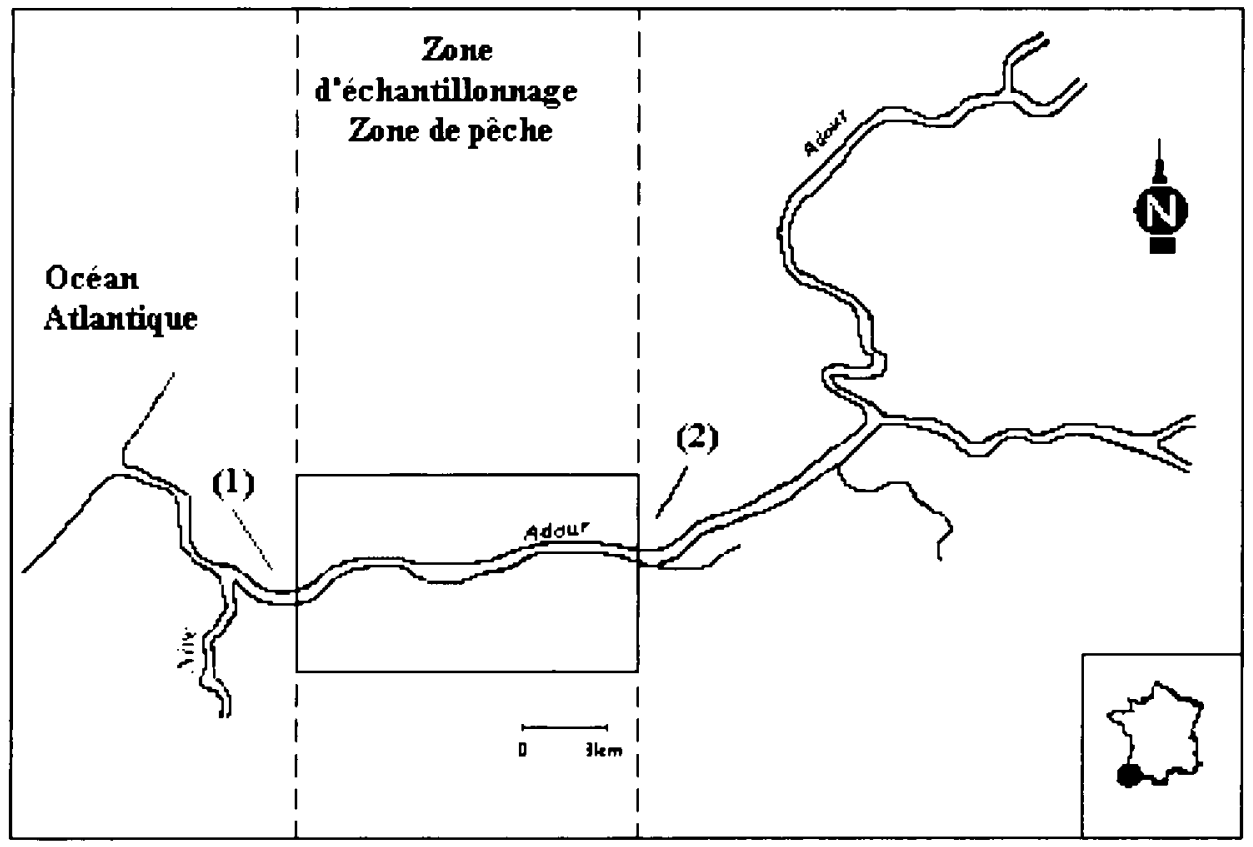

Figure 1

Localisation de l'estuaire de l'Adour et présentation de la zone prospectée. (1) Pont de l'autoroute (Bayonne) ; (2) Pont d'Urt.

\section{Figure 1}

Adour estuary and sampling area. (1) Motorway's bridge (Bayonne) ; Urt's bridge.

L'échantillonnage se pratique dans la partie maritime de l'estuaire à $10 \mathrm{~km}$ de l'embouchure et sur une distance de $12 \mathrm{~km}$ vers l'amont. Les stations prospectées se localisent entre le pont de l'autoroute au niveau de Bayonne et le pont d'Urt (à $22 \mathrm{~km}$ de la mer) (Figure 1). La zone échantillonnée se trouve en amont du front de salinité, dans un secteur de propagation de la marée dynamique. La masse d'eau est homogène aussi bien du point de vue de la salinité que de la température. La vitesse de propagation de la marée dynamique varie en fonction de l'intensité de la marée et du débit fluvial. Lors de fortes crues, le débit fluvial empêche toute intrusion saline dans l'estuaire. 
On constate également l'absence de propagation de marée dynamique et le volume d'eau en transit vers l'océan constitue un véritable mur hydrologique (ANONYME, 1993 ; DE CASAMAJOR, 1998).

\section{Collecte des données}

La campagne se déroule au rythme de la saison de migration, de novembre à mars. Le rythme des sorties est de 2 par semaine, la nuit (soit 27 sorties au total). Nous échantillonnons successivement sur 5 stations, de l'aval vers l'amont, en suivant la progression de la marée dynamique (lieu de renverse des courants) qui se propage dans l'estuaire. Sur chaque station, 3 traits de tamis d'une durée de $5 \mathrm{mn}$ sont réalisés : sur chacune des rives et au milieu de l'estuaire. Pour chaque trait de tamis, nous échantillonnons simultanément en surface et en profondeur avec 2 engins de pêche tractés par le bateau. En profondeur, on immerge un tamis circulaire d'un diamètre de 1,2 $\mathrm{m}$ : la maille du filet est de $1,6 \mathrm{~mm}$ à l'entrée et $1 \mathrm{~mm}$ au fond. En surface, le tamis utilisé est rectangulaire $(1,2 \mathrm{~m} \times 0,5 \mathrm{~m})$ avec une maille de $1 \mathrm{~mm}$. Une fermeture éclair permet la récolte des civelles après chaque trait. Quand les coefficients de marée sont supérieurs à 80 , nous utilisons 2 tamis circulaires de $0,65 \mathrm{~m}$ de diamètre pour un chalutage plus efficace face aux forts courants.

Paramètres environnementaux mesurés : les sorties se déroulent dans des conditions de débit et de coefficient de marée variables. Pour caractériser précisément le milieu de capture, nous étudions différents paramètres environnementaux. Une sonde fixée au tamis de fond mesure la température et la salinité en fonction de la profondeur pendant le trait. La localisation exacte de chaque trait de tamis est donnée par un GPS' différentiel avec une station de référence à terre permettant d'augmenter la précision du positionnement (à $1 \mathrm{~m}$ près au moins). Ce GPS est couplé à un traceur de route pour suivre la progression du bateau au cours de chaque campagne. Un sondeur à haute résolution indique en permanence la profondeur sous le bateau ce qui permet d'immerger le tamis de fond à proximité du substrat. Au cours de chaque trait un «flowmetre " mesure à l'entrée du filet de surface la vitesse du courant pour connaître le volume filtré par l'engin et déterminer ainsi la densité de civelles (en $\mathrm{g} / 100 \mathrm{~m}^{3}$ ) dans la colonne d'eau. La turbidité est mesurée, en $\mathrm{NTU}^{2}$, sur chaque station au milieu de l'estuaire en surface et en profondeur. La nébulosité et la température de l'air sont communiquées par Météo-France.

Paramètres hydrodynamiques pris en compte : le débit fluvial journalier (en $\mathrm{m}^{3} / \mathrm{s}$ ) est transmis par l'Agence de Bassin Adour-Garonne. Les mesures sont effectuées sur des stations de jaugeage situées sur l'Adour et ses principaux affluents (gave de Pau et gave d'Oloron). Le cumul des 3 valeurs donne le débit dans la zone de pêche. Les coefficients de marée sont des données publiées par le Service Hydrographique et Océanographique de la Marine (SHOM). Ils s'expriment en centièmes de l'échelle astronomique.

Calcul des poids capturés : chaque contenu de tamis est stocké dans un bocal identifié par la localisation exacte de leur provenance : station ; surface ou fond ; rive droite, milieu ou rive gauche. Le poids total capturé par trait de chalut est déterminé à partir de la pesée des civelles une par une (à $0,01 \mathrm{~g}$ près) après séchage sur papier filtre. Quand le nombre d'individus est supérieur à 50 , le surplus est pesé en une seule fois.

\section{Traitement des données et méthodes utilisées}

Les densités de civelles (en $\mathrm{g} / 100 \mathrm{~m}^{3}$ ) sont calculées pour chacun des 3 traits de tamis par station, en surface et en profondeur. La moyenne des densités, en surface $\left(D_{s}\right)$ et 
au fond $\left(D_{F}\right)$, des 3 traits est faite par station. La proportion de civelles au fond $\left(P_{F}=D_{F} / D_{T}\right)$ est exprimée en pourcentage par rapport à la densité totale $\left(D_{T}=D_{F}+D_{S}\right)$. Cet estimateur rapport permet de lisser la variabilité de l'importance des flux migratoires durant la période de migration. Ces proportions, pour chaque station, seront ensuite comparées aux conditions environnementales répertoriées durant la collecte.

La matrice des données brutes va ainsi se composer de 102 observations collectées lors de 23 sorties (sélection des sorties qui se sont déroulées dans des conditions identiques) caractérisées par des variables environnementales (Tableau I).

\section{Tableau I}

Présentation des variables environnementales lors des sorties de pêche.

\section{Table I}

\section{Environnemental factors during fishing periods.}

\begin{tabular}{ccccccc}
\hline $\begin{array}{c}\text { Date } \\
97 / 98\end{array}$ & $\begin{array}{c}\text { Débit } \\
\left(\mathrm{m}^{3} / \mathrm{s}\right)\end{array}$ & $\begin{array}{c}\text { Turbidité } \\
(\mathrm{NTU})\end{array}$ & $\begin{array}{c}\text { Nébulosité } \\
*\end{array}$ & $\begin{array}{c}\text { Température } \\
\left({ }^{\circ} \mathrm{C}\right)\end{array}$ & $\begin{array}{c}\text { Coefficient } \\
\text { de marée }\end{array}$ & $\begin{array}{c}\text { Phase lunaire } \\
\star \star\end{array}$ \\
\hline Date & Flow & Turbidity & $\begin{array}{c}\text { Cloud } \\
\text { Cover }\end{array}$ & Temperature & Tide Coeff. & Moon phases \\
\hline 2111 & 247 & 15 & $\mathrm{~N}$ & & 43 & $\mathrm{DQ}$ \\
2411 & 241 & 35 & $\mathrm{~N}$ & & 42 & $\mathrm{DQ}$ \\
2811 & 185 & 60 & $\mathrm{~N}$ & 10,86 & 68 & $\mathrm{NL}$ \\
0512 & 477 & 70 & $\mathrm{D}$ & & 69 & $\mathrm{PQ}$ \\
0912 & 246 & 10 & $\mathrm{D}$ & & 61 & $\mathrm{PQ}$ \\
1112 & 284 & 10 & $\mathrm{~N}$ & 9,3 & 79 & $\mathrm{PL}$ \\
1612 & 258 & 20 & $\mathrm{D}$ & 8 & 89 & $\mathrm{PL}$ \\
1912 & 660 & 74 & $\mathrm{~N}$ & 8,49 & 62 & $\mathrm{DQ}$ \\
2312 & 964 & 129 & $\mathrm{~N}$ & 9,58 & 39 & $\mathrm{DQ}$ \\
2912 & 974 & 177 & $\mathrm{~N}$ & 9,24 & 87 & $\mathrm{NL}$ \\
0601 & 646 & 49 & $\mathrm{D}$ & 9,92 & 59 & $\mathrm{PQ}$ \\
0801 & 429 & 22 & $\mathrm{D}$ & 8,88 & 60 & $\mathrm{PQ}$ \\
1301 & 273 & 28 & $\mathrm{~N}$ & 8,98 & 88 & $\mathrm{PL}$ \\
1601 & 350 & 92 & $\mathrm{~N}$ & 9,25 & 79 & $\mathrm{PL}$ \\
2001 & 1250 & 184 & $\mathrm{~N}$ & 10,03 & 43 & $\mathrm{DQ}$ \\
2201 & 715 & 150 & $\mathrm{~N}$ & 7,38 & 37 & $\mathrm{DQ}$ \\
2801 & 285 & 21 & $\mathrm{D}$ & 5,46 & 87 & $\mathrm{NL}$ \\
3001 & 249 & 20 & $\mathrm{D}$ & 6,2 & 106 & $\mathrm{NL}$ \\
0302 & 227 & 10 & $\mathrm{D}$ & 7,71 & 70 & $\mathrm{PQ}$ \\
0502 & 220 & 10 & $\mathrm{D}$ & 8,34 & 53 & $\mathrm{PQ}$ \\
0902 & 173 & 10 & $\mathrm{D}$ & 7,32 & 76 & $\mathrm{PL}$ \\
1302 & 158 & 10 & $\mathrm{D}$ & 8,26 & 90 & $\mathrm{PL}$ \\
2302 & 286 & 30 & $\mathrm{D}$ & 9,42 & 57 & $\mathrm{NL}$ \\
\hline & & & & & \\
\hline
\end{tabular}

* $N$ : ciel nuageux; $D$ : ciel dégagé;

** $\mathrm{PL}=$ Pleine Lune $; \mathrm{DQ}=$ Dernier Quartier $; \mathrm{NL}=$ Nouvelle Lune ; $\mathrm{PQ}=$ Premier Quartier.

${ }^{*} N$ : cloudy sky ; $D$ : clear sky.

$\star{ }^{\star *} \mathrm{PL}=$ Full Moon $; \mathrm{DQ}=$ Last Quarter $; \mathrm{NL}=$ New Moon $; \mathrm{PQ}=$ First Quarter. 
Données utilisées : l'analyse porte essentiellement sur l'influence de la lumière sur le comportement des civelles. Aussi, les variables primaires que nous considérerons sont : la turbidité, la phase lunaire et la proportion de civelles présentes au fond. Les autres variables seront utilisées uniquement à titre illustratif pour essayer dexpliquer les écarts par rapport à notre hypothèse de base : migration principalement au fond dès que la lumière pénètre dans la colonne d'eau, vis-à-vis du comportement lucifuge de la civelle.

Compte tenu de la nature discrète ou qualitative de certaines données (phase lunaire), des effets non linéaires pressentis de certaines variables (effets de seuil : turbidité) et de l'imprécision de l'estimateur rapport $\left(P_{F}\right)$, un découpage des données en classes a été effectué (Tableau II).

\section{Tableau II}

Découpage des variables principales en classes.

Table II

Factors modalities classification.

\begin{tabular}{|c|c|c|c|c|}
\hline Variable & Modalités & $\begin{array}{l}\text { Intervalle de } \\
\text { classe }\end{array}$ & Abréviation & Effectif \\
\hline \multirow[t]{4}{*}{ Phases lunaires } & Pleine Lune & $+/-3$ à 4 jours & PL & 28 \\
\hline & Dernier Quartier & autour du & DQ & 27 \\
\hline & Nouvelle Lune & changement de & NL & 19 \\
\hline & Premier Quartier & lune & $P Q$ & 28 \\
\hline \multirow{4}{*}{$\begin{array}{l}\text { *Turbidité } \\
\text { (NTU) }\end{array}$} & Turbidité Faible & de 10 à 12 & TFA & 26 \\
\hline & Turbidité Moyenne 1 & de 12 à 40 & TM1 & 36 \\
\hline & Turbidité Moyenne 2 & de 40 à 80 & TM2 & 19 \\
\hline & Turbidité Forte & de 80 à 184 & TFO & 21 \\
\hline \multirow{2}{*}{$\begin{array}{c}{ }^{\star \star} \text { Turbidité } \\
\text { (NTU) }\end{array}$} & Turbidité Faible & de 10 à 40 & TFA & 62 \\
\hline & Turbidité Forte & de 40 à 184 & TFO & 40 \\
\hline \multirow{3}{*}{$\begin{array}{c}P_{F} \\
\text { densité fond } \\
\text { (en \% du total) }\end{array}$} & Densité Faible & $<$ à $30 \%$ & DFA & 25 \\
\hline & Densité Moyenne & 30 à $70 \%$ & DMO & 30 \\
\hline & Densité Forte & $>70 \%$ & DFO & 47 \\
\hline
\end{tabular}

* découpage utilisé pour l'AFCM ; ** découpage utilisé pour le modèle loglinéaire.

${ }^{\star}$ classification for AFCM ; ${ }^{\star *}$ classification for loglinear model.

$\mathrm{PL}=$ Pleine Lune $; \mathrm{DQ}=$ Dernier Quartier $; \mathrm{NL}=$ Nouvelle Lune $; \mathrm{PQ}=$ Premier Quartier ; TFA $=$ Turbidité Faible $;$ TM1 $=$ Turbidité faible à Moyenne $: T M 2=$ Turbidité Moyenne à Forte $;$ TFO = Turbidité Forte $;$ DFO = Densité Faible $;$ DMO = Densité Moyenne : DFO = Densité Forte.

$\mathrm{PL}=$ Full Moon $; \mathrm{DQ}=$ Last Quarter $; \mathrm{NL}=$ New Moon $; \mathrm{PQ}=$ First Quarter $;$ TFA $=$ Low Turbidity; $\mathrm{TM} 1=$ Low at Median Turbidity; TM2 = Median at High Turbidity - High Turbidity ; DFO = Low Density $;$ DMO = Median Density ; DFO = High Density. 
Pour la turbidité, le seuil de 40 NTU correspond à la différence entre une eau pratiquement claire et une eau trouble à très opaque. Pour $P_{F}$, le découpage tient compte à la fois de la structure de l'histogramme de la distribution des valeurs et évite un déséquilibre trop important de l'effectif des classes. Pour l'Analyse Factorielle des Correspondances Multiples uniquement, la variable turbidité est subdivisée en 4 modalités pour éviter de donner un poids trop important aux modalités des phases lunaires (au nombre de 4). Le découpage en 4 modalités de la turbidité ne se justifie pas pour l'application du modèle linéaire généralisé.

Ainsi, le tableau à analyser se composera de 102 lignes et de 9 ou 11 colonnes qui représentent les modalités des 3 variables (Tableau II).

\section{MÉTHODOLOGIE}

Étude des covariations entre variables : une Analyse Factorielle des Correspondances Multiples (BENZECRI, 1979) est réalisée à partir de la matrice 102 lignes et 11 colonnes qui correspondent à un ensemble de valeurs ordinales représentant les modalités des variables (Tableau II). On effectue une double analyse en composantes principales sur les lignes puis sur les colonnes (valeurs pondérées par le total des lignes).

Cette technique présente l'avantage de s'appliquer aussi bien à des données qualitatives que quantitatives. Elle permet de décrire des associations ou covariations entre les 3 variables choisies (phase lunaire, turbidité et densité fond). Ces variables n'ayant pas le même statut, nous avons choisi de prendre, comme variable supplémentaire : la proportion de civelles au fond $\left(P_{F}\right)$ de manière à la projeter sur les plans factoriels formés par les 2 autres variables considérées comme actives. De cette manière, nous visualiserons mieux l'influence de la phase lunaire et de la turbidité, considérées ici comme variables explicatives, sur la densité de fond assimilée à la variable expliquée.

Étude des interactions : afin de mieux visualiser les interactions entre les 3 variables, un graphique des interactions est effectué en considérant les variables : turbidité et phase lunaire comme des facteurs et la variable $P_{F}$ comme variable à expliquer. Nous choisissons comme facteur de base la turbidité présentant 2 niveaux (ligne des abscisses) ; comme facteur secondaire ou modulateur : la phase lunaire à 4 niveaux.

L'analyse est effectuée à partir d'une matrice de 102 lignes et 3 colonnes : les 2 premières colonnes représentant les niveaux des 2 facteurs explicatifs (turbidité et phase lunaire) et la troisième, la valeur brute de la densité de fond (en pourcentage de la densité totale).

La représentation obtenue nous donne les variations de la moyenne, de la médiane et du premier et troisième quartiles de la distribution des valeurs de $P_{F}$ en fonction des 4 niveaux du facteur secondaire, pour chaque niveau du facteur de base.

Modélisation des densités : nous construisons un tableau de contingence (Tableau III) dont chaque cellule contient le nombre d'observations pour chaque combinaison des modalités des variables : densité fond $\left(P_{f}\right)$, phase lunaire (lune) et turbidité (turb). On considère seulement 2 modalités de turbidité pour éviter un trop grand nombre de cellules aux effectifs nuls dans le tableau de contingence. Les tableaux marginaux (Tableaux IVa et IVb) rendent compte des associations entre les variables $P_{F}$ et lune d'une part, et $P_{F}$ et turb d'autre part. 


\section{Tableau III}

Observations par combinaison des modalités des variables densités fond-totale $\left(P_{F}\right)$, phase lunaire (lune) et turbidité (turb).

\section{Table III}

Observations for the combination of bottom/total densities $\left(\boldsymbol{P}_{F}\right)$, rate of moon phases (lune) and turbidity (turb).

\begin{tabular}{lcccc}
\hline Phase lunaire (lune) & Turbidité (turb) & \multicolumn{3}{c}{ Rapport des densités fond-totale $\left(P_{F}\right)$} \\
& & Faible & Moyen & Fort \\
\hline Pleine Lune (PL) & Faible & 0 & 2 & 21 \\
& Forte & 3 & 2 & 0 \\
\hline Dernier Quartier (DQ) & Faible & 2 & 3 & 5 \\
& Forte & 10 & 4 & 3 \\
\hline Nouvelle Lune (NL) & Faible & 4 & 8 & 0 \\
& Forte & 3 & 2 & 2 \\
\hline Premier Quartier (PQ) & Faible & 0 & 1 & 16 \\
& Forte & 3 & 8 & 0 \\
\hline
\end{tabular}

\section{Tableau IV}

Observations par combinaison des modalités des variables rapport des densités fond-surface $\left(P_{F}\right)$ et a) turbidité (turb) ; b) phase lunaire (lune).

\section{Table IV}

Observations for the combination of bottom/surface densities $\left(\boldsymbol{P}_{F}\right)$ and rate of a) turbidity (turb) ; b) moon phase (Iune).

\begin{tabular}{|c|c|c|c|}
\hline & \multicolumn{3}{|c|}{ Rapport des densités fond-totale $\left(P_{F}\right)$} \\
\hline & Faible & Moyen & Fort \\
\hline \multicolumn{4}{|c|}{ a) turbidité } \\
\hline Faible & 6 & 14 & 42 \\
\hline Forte & 19 & 16 & 5 \\
\hline \multicolumn{4}{|c|}{ b) phase lunaire } \\
\hline Pleine Lune (PL) & 3 & 4 & 21 \\
\hline Dernier Quartier (DQ) & 12 & 7 & 8 \\
\hline Nouvelle Lune (NL) & 7 & 10 & 2 \\
\hline Premier Quartier (PQ) & 3 & 9 & 16 \\
\hline
\end{tabular}

Les modèles utilisés sont de type loglinéaire. La loi de distribution choisie pour les comptages est celle de Poisson et la fonction de lien qui décrit comment la moyenne dépend des prédicteurs linéaires est le Logarithme. Cette technique d'analyse a déjà été utilisée pour mesurer l'influence des conditions hydroclimatiques sur l'abondance des CPUE d'Aloses (PROUZET et al., 1994 ; BADIA et PROUZET, 1996). 
Notations : pour le Tableau IV, nous adoptons les notations suivantes : $i$ est l'indice ligne, $j$ est l'indice colonne ; pour le Tableau IVa : $i(=2)$ le nombre de lignes et $j(=3)$ le nombre de colonnes; pour le Tableau IVb : $i(=4)$ le nombre de lignes et $j(=3)$ le nombre de colonnes. Les $n(102)$ observations de l'échantillon sont réparties dans les $n=i j(=6$ pour le Tableau IVa ou = 12 pour le Tableau IVb) cellules du tableau. L'effectif de chaque cellule $i j$ est $n_{i j}$; il y a $n_{i+}$ observations dans la ligne $i$ et $n_{+j}$ dans la colonne $j$.

Dans le cas d'un échantillon multinômial, les probabilités $\left\{\pi_{i j}\right\}$ des cellules $\{i j\}$ représentent des réponses par modalité de la variable facteur. Si les observations sont indépendantes $\left(\pi_{i j}=\pi_{i t} \pi_{t j}\right)$, les effectif́s espérés $\left(m_{i j}=n \pi_{i j}\right)$ sont égaux à $m_{i j}=n \pi_{i+} \pi_{+j}$ pour tout $i$ et $j$.

Sur l'échelle logarithmique, l'indépendance s'exprime sous une forme additive et telle que : $\log m_{i j}=\log n+\log \pi_{i+}+\log \pi_{+j}$. Si l'on note $X$ et $Y$ les variables ligne et colonne, l'équation précédente est équivalente à :

$$
\log m_{i j}=\mu+\lambda_{1}^{x}+\lambda_{1}^{r}
$$

avec $\mu$ le paramètre général, $\lambda_{1}^{\times}$le paramètre associé à la modalité $i$ de la variable $X$ et $\lambda_{1}^{r}$ celui associé à la modalité $j$ de la variable $Y$. Pour des problèmes d'identification, ces paramètres doivent satisfaire à des contraintes telles que :

$$
\sum_{i} \lambda_{i}^{x}=\sum_{i} \lambda_{j}^{Y}=0
$$

S'il existe une dépendance entre les variables $X$ et $Y$, on introduit un terme d'interaction, le modèle s'écrit :

$$
\log m_{i j}=\mu+\lambda_{i}^{X}+\lambda_{j}^{Y}+\lambda_{i j}^{X Y} \quad \forall_{,}
$$

Les nouveaux paramètres $\lambda_{11}^{\text {rr }}$ mesurent les écarts à l'indépendance des variables $X$ et $Y$. Ils constituent des paramètres d'association. Pour être identifiables, ils satisfont également à des contraintes telles que :

$$
\forall_{i} \sum_{j} \lambda_{i j}^{. Y \gamma}=0 \text { et } \forall_{j} \sum_{i} \lambda_{i j}^{\lambda Y}=0
$$

On considère maintenant les observations du Tableau I relatives aux variables $X$ (turbidité), $Y$ (phase lunaire) et $Z\left(P_{F}\right)$. On peut tester les 3 modèles suivants :

a) si les 3 variables sont mutuellement indépendantes $\left(\pi_{t, k}=\pi_{1, \ldots} \pi_{\ldots, \ldots} \pi_{\ldots,}\right.$ pour tout $i, j$ et $k$ ), le modèle d'indépendance mutuelle s'écrit alors :

$$
\log m_{i j k}=\mu+\lambda_{i}^{X}+\lambda^{Y}+\lambda_{k}^{Z}
$$

b) si la variable $Z$ est indépendante de $X$ et de $Y\left(\pi_{i k}=\pi_{i, j} \pi_{\ldots, k}\right.$ pour tout $i, j$ et $\left.k\right)$, le modèle d'indépendance jointe s'écrit :

$$
\log m_{i j k}=\mu+\lambda_{i}^{X}+\lambda_{j}^{Y}+\lambda_{k}^{Z}+\lambda_{i j}^{X Y}
$$


c) si $X$ et $Y$ sont indépendantes dans le tableau partiel associé à la modalité $k$ de $Z$, $X$ et $Y$ sont dites conditionnellement indépendantes au niveau $k$ de $Z$. Comme $\pi_{1}=\pi_{1 .} \pi_{1}$ pour tout $i$ et $j$ avec $\pi_{1 .}=\pi, \pi$, la distribution conjointe de $X$ et de $Y$ au niveau $k$ de $Z$, le modèle d'indépendance conditionnelle de $X$ et de $Y$ s'écrit :

$$
\log m_{t i k}=\mu+\lambda_{i}^{i}+\lambda_{1}^{Y}+\lambda_{k}^{L}+\lambda_{i k}^{M z}+\lambda_{i h}^{3}
$$

Pour les tableaux à 3 dimensions:

$$
\log m_{i j k}=\mu+\lambda_{i}^{Y}+\lambda_{i}^{Y}+\lambda_{k}^{\prime}+\lambda_{i j}^{Y y}+\lambda_{i k}^{Y /}+\lambda_{i k}^{Y /}
$$

est le modèle loglinéaire le plus général qui puisse être analysé.

Pour vérifier le bon ajustement de ces modèles aux observations, on utilise la "fonction de déviance » qui est reliée linéairement au logarithme de la vraisemblance et qui est utilisée comme critère d'ajustement. A l'instar du numérateur du test de Fisher, la différence de déviance entre 2 modèles mesure la contribution des paramètres pour lesquels ils diffèrent. Dans la théorie asymptotique, sous l'hypothèse nulle que le plus petit modèle est correct, la différence de déviance suit une loi du $\chi^{2}$ avec comme degrés de liberté la différence de degrés de liberté entre les 2 modèles.

Afin de voir quel est l'apport des facteurs et de leurs interactions sur l'ajustement (introduction) ou sur la dégradation (retrait) du modèle, nous utilisons une procédure pas à pas dont les bornes sont comprises entre le modèle minimum et le modèle saturé. Le choix se fait d'après le critère statistique d'Akaike : $A I C=D+2 p \Phi$ (CHAMBERS et HASTIE, 1991 ) avec $D$ : déviance expliquée par le facteur incorporé ou par l'interaction, $p$ le nombre de degrés de liberté utilisé pour l'ajustement et $\Phi$ l'estimation du paramètre de dispersion qui pour la loi de Poisson est égal à 1.

\section{RÉSULTATS}

\section{Conditions environnementales au moment des captures}

Les conditions environnementales varient d'une sortie de pêche à l'autre. Les débits suivent les mêmes tendances que la turbidité. La turbidité augmente et diminue simultanément aux variations des débits fluviaux. Mais cette variable intervient également sur les mouvements horizontaux des civelles, c'est pourquoi nous ne la prenons pas en considération pour étudier les fluctuations verticales des densités. Le coefficient de marée est déterminé par le cycle lunaire, les forts coefficients correspondant aux périodes de pleine et nouvelle lune et les faibles coefficients aux premier et dernier quartiers (Tableau II). La phase lunaire et la turbidité ne sont pas des variables environnementales corrélées ; cependant, on constate que les sorties en dernier quartier se font par ciel couvert, alors que les sorties lors de premier quartier se font par ciel dégagé (Tableau II). Les fluctuations de température sont faibles au cours de la campagne de pêche et sont homogènes dans la colonne d'eau. On note cependant une diminution des températures fin janvier. La salinité reste nulle au cours de la sortie.

\section{Analyse descriptive des relations entre densités, turbidité et cycle lunaire}

L'analyse des valeurs propres et du pourcentage de variance expliqué par les axes factoriels montre que l'inertie du nuage de points est forte sur le premier axe, puis diminue de façon sensiblement équivalente sur les 2 autres axes. Les 3 premiers axes expliquent $69 \%$ de l'inertie totale du nuage de points qui est estimée à 3 (somme totale des valeurs propres). 


\section{Tableau V}

Variances sur les axes factoriels principaux et contributions à l'inertie totale.

\section{Table V}

Variances on principal factorial axes and contributions to total inertia.

\begin{tabular}{cccc}
\cline { 2 - 4 } & $\mathbf{1}$ & AXES \\
& $\mathbf{1}$ & $\mathbf{2}$ & $\mathbf{3}$ \\
\hline Val. Propres & 0,79 & 0,68 & 0,61 \\
Contribution $\%$ & 26 & 23 & 20 \\
(Val. Propres) $1 / 2$ & 0,89 & 0,82 & 0,78 \\
\hline
\end{tabular}

\section{Tableau VI}

Contributions des modalités des variables à l'inertie totale.

\section{Table VI}

Contributions to total inertia.

\begin{tabular}{rccc} 
& \multicolumn{3}{c}{ AXES } \\
\cline { 2 - 4 } & $\mathbf{1}$ & $\mathbf{2}$ & $\mathbf{3}$ \\
\hline PL & 4,7 & 31,6 & 0 \\
DQ & 17,9 & 0,8 & 18,1 \\
NL & 8,4 & 0,6 & 31,7 \\
PQ & 19,1 & 17,1 & 0,1 \\
TFA & 27,8 & 8,5 & 1 \\
TM1 & 5,6 & 0,7 & 26 \\
TM2 & 1,4 & 38,4 & 0,9 \\
TFO & 15,1 & 2,4 & 22,2 \\
\hline Turbidité & $\mathbf{1 0 0}$ & $\mathbf{1 0 0}$ & $\mathbf{1 0 0}$ \\
\hline
\end{tabular}

$\mathrm{PL}=$ Pleine Lune $; \mathrm{DQ}=$ Dernier Quartier $; \mathrm{NL}=$ Nouvelle Lune $; \mathrm{PQ}=$ Premier Quartier ; TFA $=$ Turbidité Faible $; \mathrm{TM} 1=$ Turbidité Faible à Moyenne $; \mathrm{TM} 2=$ Turbidité Moyenne à Forte ; TFO = Turbidité Forte.

$P L=$ Full Moon $; \mathrm{DQ}=$ Last Quarter $; \mathrm{NL}=$ New Moon $; \mathrm{PQ}=$ First Quarter $; \mathrm{TFA}=\mathrm{Low}$ Turbidity $; \mathrm{TM} 1=$ Low at Median Turbidity $; \mathrm{TM} 2=$ Median at High Turbidity $; \mathrm{TFO}=$ High Turbidity. 


\section{Étude des variables}

L'axe 1 discrimine bien toutes les variables alors que l'axe 2 caractérise principalement les phases lunaires (Figure 2). L'analyse des associations entre les variables sur le premier plan factoriel (Figure 2) montre que les densités fortes en profondeur (DFO) sont associées aux turbidités faibles (TFA) surtout autour de la pleine lune $(P L)$ et du premier quartier $(P Q)$. A l'inverse, les densités faibles en profondeur (DFA) sont fortement associées aux fortes turbidités (TFO) au cours du dernier quartier (DQ). La nouvelle lune (NL) et les densités moyennes (DMO) sont moins bien associées aux modalités de turbidité sur ce plan factoriel. Les turbidités moyennes (TM1 et TM2) sont peu associées aux autres modalités.

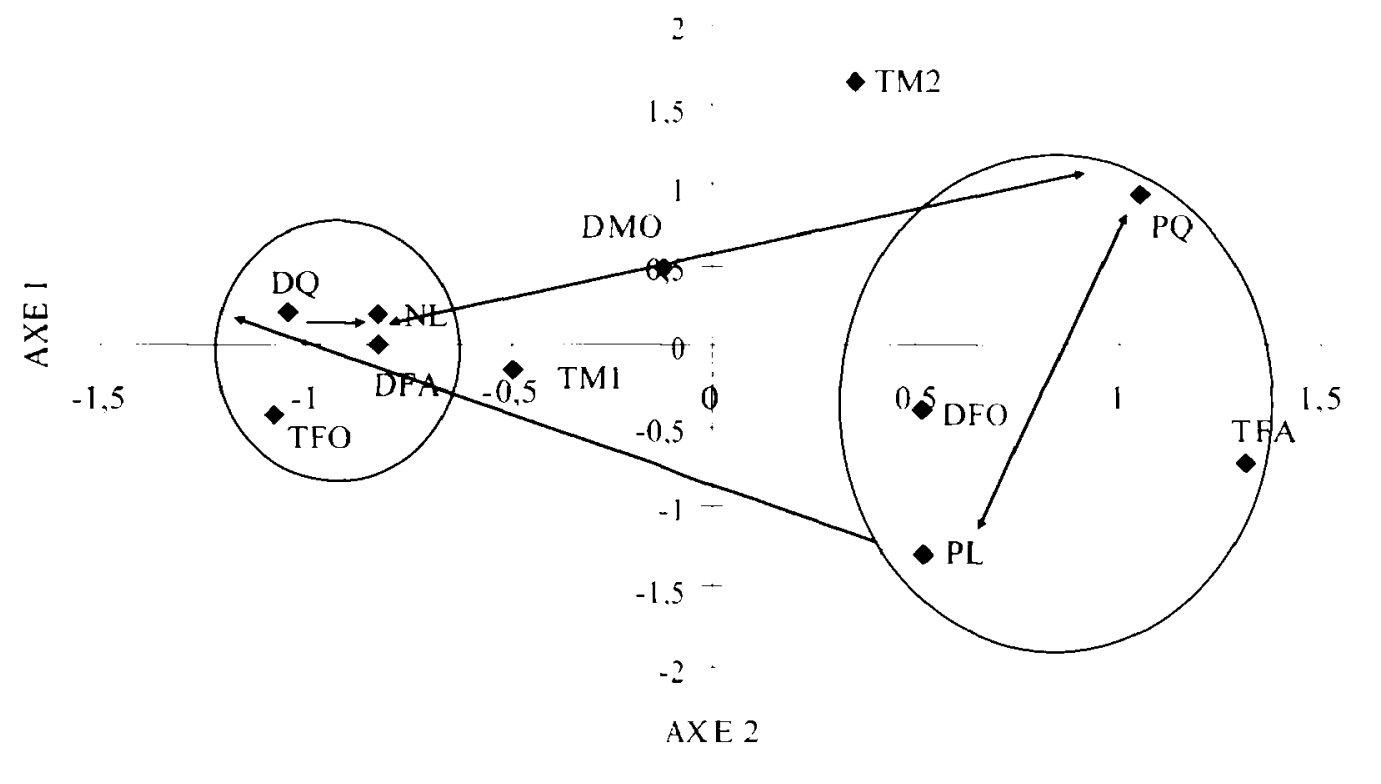

$\mathrm{PL}=$ Pleine Lune $; \mathrm{DQ}=$ Dernier Quartier $; \mathrm{NL}=$ Nouvelle Lune $; \mathrm{PQ}=$ Premier Quartier TFA $=$ Turbidité Faible $; M T 1=$ Turbidité Faible à Moyenne $; \mathrm{TM} 2=$ Turbidité Moyenne à Forte $;$ TFO = Turbidité Forte $;$ DFA $=$ Densité Faible $;$ DMO = Densité Moyenne ; DFO = Densité Forte.

PL $=$ Full Moon $; \mathrm{DQ}=$ Last Quarter $; \mathrm{NL}=$ New Moon $; \mathrm{PQ}=$ First Quarter $; \mathrm{TFA}=$ Low Turbidity; $M T 1=$ Low and Median Turbidity; TM2 = Median at High Turbidity; TFO = High Turbidity ; DFO = Low Density $; \mathrm{DMO}=$ Median Density $; \mathrm{DFO}=$ High Density.

\section{Figure 2}

Positions des différentes modalités des variables dans le premier plan factoriel.

\section{Figure 2}

\section{Distribution of variables in the first factorial plane.}


L'axe 3 discrimine bien les modalités des phases lunaires (Tableau VI et Figure 3). Nous observons des associations entre les densités fortes en profondeur (DFO) et la turbidité faible et moyenne (TFA et TM2) au moment de la pleine lune (PL) et du premier quartier (PQ). Ces associations s'opposent à celles du dernier quartier (DQ) avec les densités faibles (DFA) et à forte turbidité (TFO). La nouvelle lune (NL) est associée aux densités moyennes (DMO) en profondeur et aux turbidités moyennes (TM1).

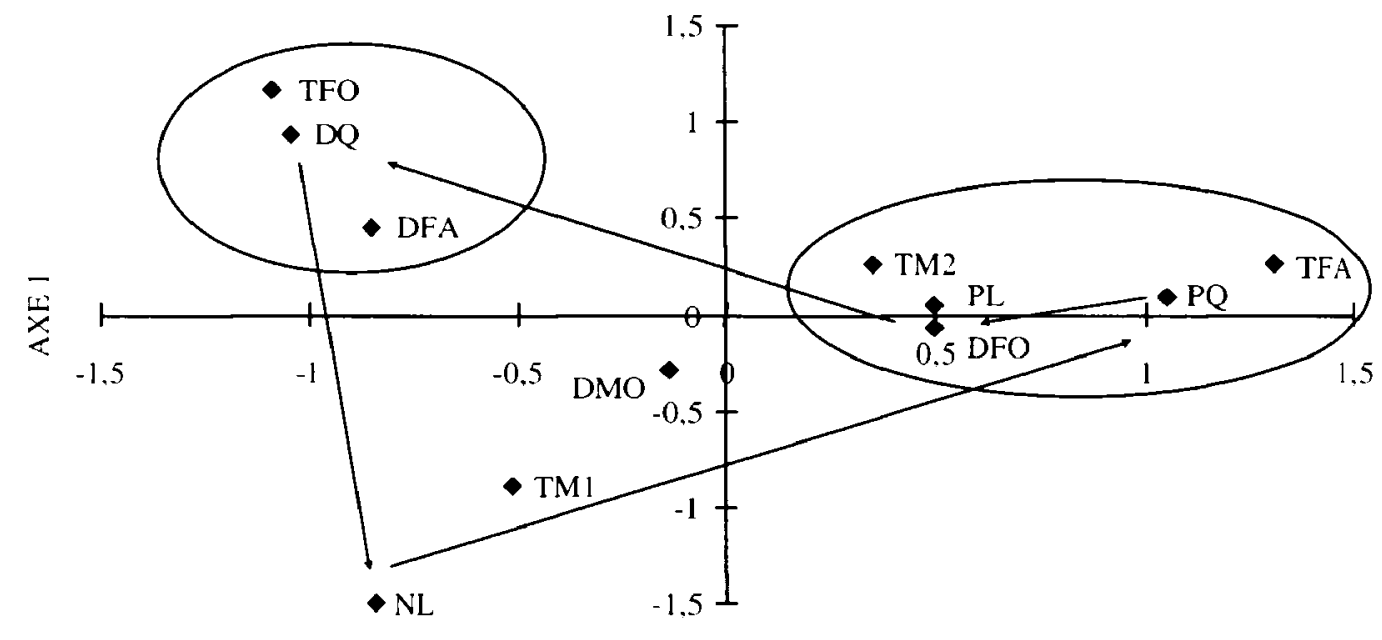

AXE 3

$\mathrm{PL}=$ Pleine Lune $; \mathrm{DQ}=$ Dernier Quartier $; \mathrm{NL}=$ Nouvelle Lune $; \mathrm{PQ}=$ Premier Quartier TFA $=$ Turbidité Faible $;$ MT1 = Turbidité Faible à Moyenne $;$ TM2 $=$ Turbidité Moyenne à Forte $;$ TFO = Turbidité Forte $;$ DFA $=$ Densité Faible $;$ DMO = Densité Moyenne ; DFO = Densité Forte.

$\mathrm{PL}=$ Full Moon $; \mathrm{DQ}=$ Last Quarter $; \mathrm{NL}=$ New Moon $; \mathrm{PQ}=$ First Quarter $;$ TFA $=$ Low Turbidity; MT1 = Low and Median Turbidity; TM2 = Median at High Turbidity; TFO = High Turbidity ; DFO = Low Density ; DMO = Median Density ; DFO = High Density.

\section{Figure 3}

Positions des différentes modalités des variables dans le second plan factoriel.

Figure 3

Distributions of variables in the second factorial plane.

\section{Visualisation des interactions}

L'examen des dispersions des modalités des phases lunaires et de turbidité précise les tendances observées par l'analyse multivariée. Les associations sur les plans factoriels sont confirmées par les dispersions des proportions de densités fond (Figure 4). La turbidité apparaît sur ces graphiques comme le facteur entraînant la plus grande variabilité au sein des données. 2 classes de turbidité (TFA et TFO) sont conservées en raison des faibles associations observées entre les 2 modalités intermédiaires (TM1 et TM2) et les modalités de densités. 


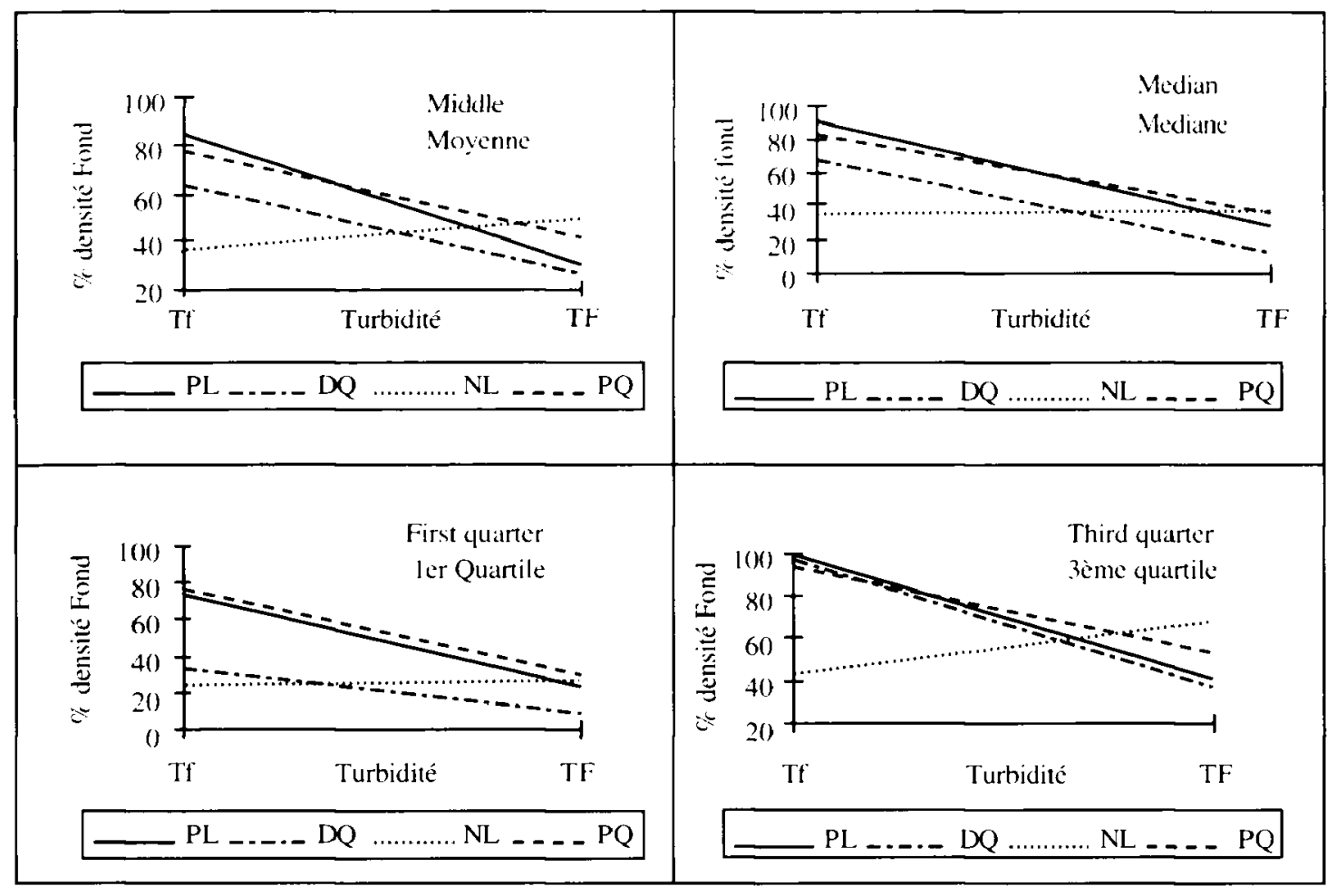

$\mathrm{PL}=$ Pleine Lune $; \mathrm{DQ}=$ Dernier Quartier $; \mathrm{NL}=$ Nouvelle Lune $; \mathrm{PQ}=$ Premier Quartier.

$P L=$ Full Moon ; $D Q=$ Last Quarter $; \mathrm{NL}=$ New Moon ; $P Q=$ First Quarter.

\section{Figure 4}

Dispersion de $\boldsymbol{P}_{F}$ (proportion densité fond/densité totale) en fonction des phases lunaires et des turbidités ( $\mathrm{Tf}=$ Turbidité faible et TF $=$ Turbidité forte).

\section{Figure 4}

Dispersion of variable ( $\boldsymbol{P}_{F}$ in \% bottom densities) according to moon phases and turbidity levels (Tf = low turbidity and TF = high turbidity).

La pente de la droite montre qu'en nouvelle lune (NL), le pourcentage de densité fond reste relativement constant, quel que soit le niveau de turbidité. La variabilité est plus marquée pendant les autres phases lunaires. En pleine lune (PL) et en premier quartier $(P Q)$, on constate une forte dispersion des données en fonction de la turbidité (Figure 4). Les densités au fond sont relativement plus fortes par faible turbidité alors qu'elles diminuent par forte turbidité. Le dernier quartier (DQ) se différencie de la pleine lune (PL) et du premier quartier (PQ) par une plus faible dispersion des données (Figure 4). La luminosité nocturne est équivalente pendant le premier et le dernier quartiers, or la dispersion des données est plus faible durant le dernier quartier (DQ). Cette différence peut être expliquée par la nébulosité, les sorties durant le dernier quartier (DQ) ont lieu par ciel couvert alors qu'en premier quartier (PQ) le ciel est dégagé (Tableau VII). 


\section{Tableau VII}

Classement des sorties en fonction de la nébulosité et de la phase lunaire.

\section{Table VII}

Distribution of the sampling periods according to cloud cover and moon phases.

\begin{tabular}{ccccc}
\hline Sorties & $\mathrm{PL}$ & $\mathrm{DQ}$ & $\mathrm{NL}$ & $\mathrm{PQ}$ \\
\hline Ciel nuageux & 3 & 6 & 2 & 0 \\
Ciel dégagé & 3 & 0 & 3 & 6 \\
\hline
\end{tabular}

$\mathrm{PL}=$ Pleine Lune $; \mathrm{DQ}=$ Dernier Quartier $; \mathrm{NL}=$ Nouvelle Lune $; \mathrm{PQ}=$ Premier Quartier.

$\mathrm{PL}=$ Full Moon $; \mathrm{DQ}=$ Last Quarter $; \mathrm{NL}=$ New Moon $; \mathrm{PQ}=$ First Quarter.

\section{Quantification des interactions et modélisation du tableau de contingence}

Interaction entre turbidité et proportion de civelles au fond (Tableau VIII)

\section{Tableau VIII}

Analyse des déviances pour le modèle $\left(\sim P_{F}+\right.$ turb $\left.+P_{F}^{*} t u r b\right)$.

\section{Table VIII}

Deviances analysis for the model $\left(\sim P_{F}+\right.$ turb $\left.+P_{F}{ }^{\star} t u r b\right)$.

\begin{tabular}{cccccc}
\hline Facteurs & Deg. liberté & Déviance & $\begin{array}{c}\text { Deg. Liberté } \\
\text { résiduels }\end{array}$ & $\begin{array}{c}\text { Déviance } \\
\text { résiduelle }\end{array}$ & $P($ Chi2 $)$ \\
\hline Constante & & & 5 & 48,09 & \\
$P_{F}$ & 2 & 7,55 & 3 & 40,54 & 0,02 \\
turb & 1 & 4,78 & 2 & 35,75 & 0,03 \\
$P_{F}^{*}$ turb & 2 & 35,75 & 0 & 0 & $2 . \mathrm{E}(-8)$ \\
\hline
\end{tabular}

Le terme d'interaction $P_{F}+$ turb explique une grande part de la déviance du modèle. Cette différence de déviance entre le modèle simple (termes indépendants et interaction turb*lune) et le modèle complet est très significative (Tableau VIII). Cela indique clairement qu'il y a des interactions très fortes entre les modalités de la turbidité et la variable $P_{F}$. L'analyse du tableau de contingence indique bien que cette interaction est positive : augmentation de la proportion de civelles en profondeur quand la turbidité augmente. 
Interaction entre phase lunaire et proportion de civelles au fond (Tableau IX)

Tableau IX

Analyse des déviances pour le modèle $\left(\sim P_{F}+\right.$ lune $\left.+P_{F}{ }^{\star} l u n e\right)$.

Table IX

Deviances analysis for the model $\left(\sim P_{F}+/\right.$ Iune $\left.+P_{F}^{\star} / u n e\right)$.

\begin{tabular}{cccccc}
\hline Facteurs & Deg. Liberté & Déviance & $\begin{array}{c}\text { Deg. Liberté } \\
\text { résiduels }\end{array}$ & $\begin{array}{c}\text { Déviance } \\
\text { résiduelle }\end{array}$ & $P(\mathrm{Chi})$ \\
\hline Constante & & & 11 & 40,06 & \\
$P_{F}$ & 2 & 7,55 & 9 & 32,51 & 0,023 \\
lune & 3 & 2,38 & 6 & 30,13 & 0,497 \\
$P_{F}^{*} /$ lune & 6 & 30,13 & 0 & 0 & $0,37 . \mathrm{E}(-4)$ \\
\hline
\end{tabular}

On note également une interaction très significative entre la proportion de civelles présentes au fond et la phase lunaire $P_{\mathrm{f}}+$ lune.

\section{Choix du modèle}

Le modèle minimum représente les termes indépendants du modèle $\left(P_{F}+\right.$ turb + lune) et le terme d'interaction des 2 facteurs explicatifs (turb*/une) de la variable $P_{F}$. La sélection des termes du modèle linéaire généralisé se fait à l'aide d'une méthode pas à pas qui part du modèle minimal suivant :

$$
F_{i l} \sim P_{F}+\text { turb + lune + turb*lune }
$$

avec $F_{l}$ : fréquence de la cellule ij du tableau de contingence.

La procédure pas à pas nous permet de considérer les parts de déviance qui sont apportées par l'incorporation des différentes interactions d'ordre 2 ou d'ordre 3 (Tableau X).

\section{Tableau $X$}

Parts de déviance allouées aux interactions d'ordre 2 et d'ordre 3.

\section{Table $\mathrm{X}$}

Level deviance in the model linked to order 2 and 3 interactions.

\begin{tabular}{cccccc}
\hline Etape & Deg. Liberté & Déviance & $\begin{array}{c}\text { Deg. Liberté } \\
\text { résiduels }\end{array}$ & $\begin{array}{c}\text { Déviance } \\
\text { résiduelle }\end{array}$ & AIC \\
\hline $\begin{array}{c}\text { Modèle } \\
\text { minimum }\end{array}$ & 2 & 35,7 & 14 & 91,47 & 111,47 \\
$\begin{array}{c}\text { Interaction } \\
P_{F}^{*} \text { turb }\end{array}$ & 6 & 26,1 & 6 & 55,8 & 79,9 \\
$\begin{array}{c}\text { Interaction } \\
P_{F}^{*} \text { lune } \\
\text { Interaction }\end{array}$ & 6 & 29,7 & 0 & 29,7 & 65,7 \\
$P_{F}^{*}$ turb*lune & 6 & 12 & 0 & 48 \\
\hline
\end{tabular}


Le Tableau $X$ indique bien que la part la plus forte de déviance expliquée est liée aux interactions entre $P_{F}$ et turb d'une part, et $P_{F}$ et lune d'autre part. Ces interactions d'ordre 2 participent à $56 \%$ de la déviance totale du tableau de contingence. La déviance liée à l'interaction d'ordre 3 confirme bien les forts liens existant entre la proportion de civelles au fond et le couple turbidité-phase lunaire.

L'analyse des déviances et de l'évolution du critère AIC (Tableau X) nous permet de choisir un modèle reproduisant une grande part de la déviance totale :

$$
F_{i j} \sim \frac{P_{F}+\text { turb }+ \text { lune }+ \text { turb*lune }}{\text { Modèle minimum }}+P_{F}^{*} \text { turb }+P_{F}^{*} / u n e .
$$

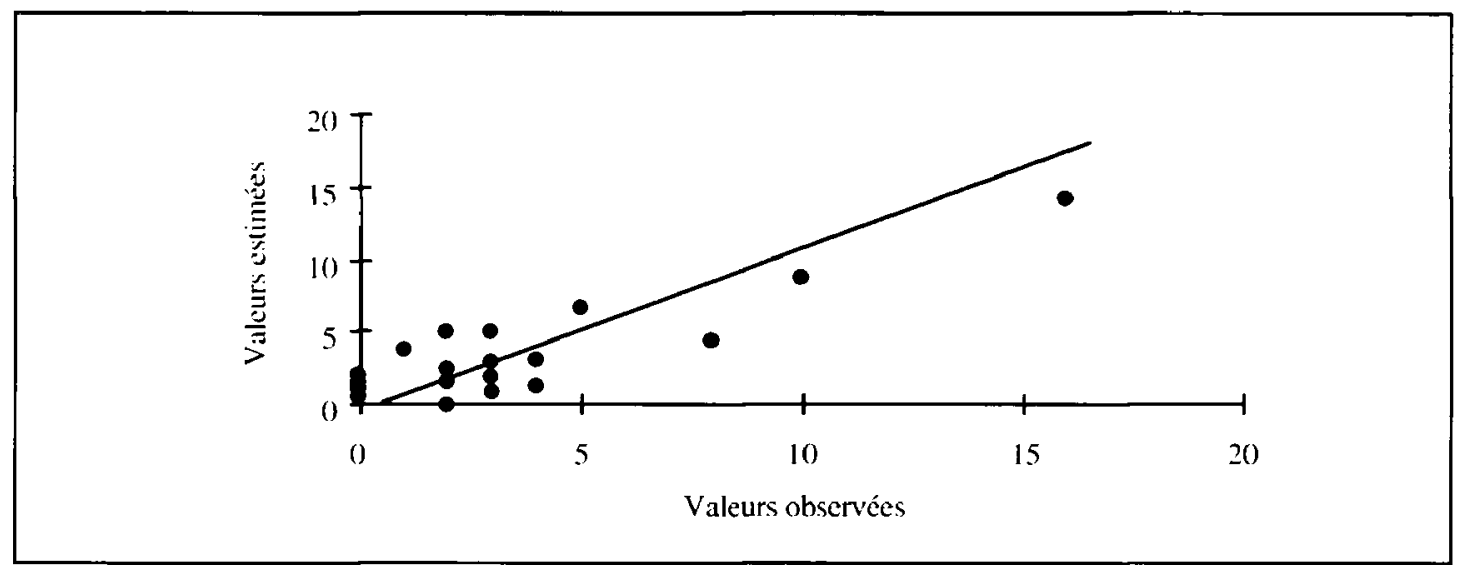

$$
\left(\sim P_{F}+\text { turb }+ \text { lune }+ \text { turb }{ }^{*} \text { lune }+P_{F}^{*} \text { turb }+P_{F}^{*} \text { lune }\right)
$$

\section{Figure 5}

Représentation des valeurs observées en fonction des valeurs estimées par le modèle.

\section{Figure 5}

Observed values according to estimated values by the model.

La Figure 5 montre que les données estimées reproduisent correctement la matrice des données observées avec simplement la prise en compte du modèle minimum et de 2 interactions d'ordre 2.

La déviance résiduelle est de 29,74 et le Tableau XI précise les résultats de l'analyse de déviance.

\section{DISCUSSION - CONCLUSION}

Cette campagne d'échantillonnage est mise en place pour étudier le comportement migratoire des civelles en fonction de la variabilité des conditions environnementales. En effet, les captures professionnelles de civelles sur l'Adour ne se font qu'en surface. Ces données offrent de bonnes indications sur les mouvements migratoires en surface, mais aucune information n'est disponible sur ce qu'il se passe en profondeur. C'est pourquoi des prélèvements sont réalisés simultanément en surface et en profondeur. 


\begin{tabular}{|c|c|c|c|c|c|}
\hline \multirow[t]{2}{*}{ Facteurs } & \multirow[t]{2}{*}{ Deg. Liberté } & \multirow[t]{2}{*}{ Déviance } & \multirow{2}{*}{$\begin{array}{c}\text { Différence de } \\
\text { Deg. Liberté }\end{array}$} & \multirow{2}{*}{$\begin{array}{c}\text { Différence de } \\
\text { Déviance }\end{array}$} & \multirow[t]{2}{*}{$P($ Chi2) } \\
\hline & & & & & \\
\hline Total & & & 23 & 118,4 & \\
\hline lune & 3 & 2,38 & 20 & 110,85 & 0,497 \\
\hline$P_{F}$ & 2 & 7,56 & 18 & 106,06 & 0,022 \\
\hline Turb & 1 & 4,78 & 17 & 103,68 & 0,028 \\
\hline turb*lune & 3 & 12,21 & 14 & 91.47 & 0.0066 \\
\hline$P_{F}^{*}$ turb & 2 & 35,7 & 12 & 55,79 & 0 \\
\hline$P_{F}^{*} /$ une & 6 & 26,05 & 6 & 29,74 & $0,21 . E(-3)$ \\
\hline
\end{tabular}

L'objectif de ce travail était de caractériser les déplacements de la civelle dans la colonne d'eau en zone estuarienne et non la migration longitudinale. C'est pourquoi nous échantillonnons au moment où les courants de débit fluvial et de coefficient de marée s'annulent. Nous considérons, lors de cette campagne expérimentale, 2 facteurs environnementaux pouvant modifier l'intensité de la lumière nocturne dans la colonne d'eau : la phase lunaire et la turbidité.

\section{Relations densités-phases lunaires}

L'analyse du graphique des interactions (Figure 4) montre clairement qu'en période de nouvelle lune, quelles que soient les conditions de turbidité, les civelles sont réparties dans toute la colonne d'eau. La proportion de civelles à proximité du fond s'accroît lorsque la clarté lunaire diffuse fortement dans la masse d'eau. C'est le cas, particulièrement, quand on se trouve en période de pleine lune avec une turbidité faible.

De nombreux travaux assimilent l'influence du cycle lunaire à celui du cycle de marée (CANTRELLE, 1981 ; GASCUEL, 1987) sans considérer l'effet lumière. Les fluctuations des coefficients de marée suivent les fluctuations du cycle lunaire avec un décalage de 2 jours. Les variations du coefficient de marée (en fonction du débit fluvial) modifient la vitesse du courant. Ce paramètre influence les déplacements de civelles dans l'estuaire mais reste un facteur de capturabilité secondaire. Ce protocole d'échantillonnage permet de dissocier les facteurs environnementaux qui induisent des mouvements verticaux de migration. Ces facteurs différent de ceux qui induisent des mouvements horizontaux.

L'analyse des carnets de pêche sur l'Adour montre que l'augmentation des captures professionnelles en surface, en zone fluviale est fortement corrélée aux périodes de nouvelle lune (BRU et al., 1996 ; DE CASAMAJOR et al., 1996). Les carnets de pêche ne 
nous apportent aucune information sur les déplacements de civelles en profondeur. Nous précisons les résultats par cette campagne de pêche expérimentale grâce à la variable $P_{F}$ qui décrit également les déplacements des civelles en profondeur (Figure 4). En nouvelle lune, quand les captures en surface sur l'Adour augmentent (DE CASAMAJOR et al., 1996), les civelles sont également présentes en profondeur (Figure 4). En pleine lune, les captures tout comme les densités sont faibles en surface. Les civelles sont absentes en surface, mais les densités sont importantes en profondeur (Figure 4). Nos résultats confirment ceux de JELLYMAN (1979) sur Anguilla spp. et de TZENG (1985) sur Anguilla japonica.

\section{Relations densités et turbidité-phase lunaire}

La turbidité provoque une atténuation de la quantité de lumière pénétrant en profondeur. Elle joue un rôle prépondérant sur la localisation des civelles dans la colonne d'eau. Cela est particulièrement visible au cours de la période lunaire (pleine lune, dernier quartier et premier quartier) où la quantité de lumière est importante.

Cette hypothèse de l'importance de l'effet lumière est renforcée par l'analyse des situations en phase de premier quartier et de dernier quartier. Les réactions des civelles sont quelque peu différentes malgré une intensité lumineuse équivalente. Les observations de la nébulosité (atténuation de la clarté nocturne) montrent qu'il existe des situations différentes de couverture nuageuse entre les 2 périodes (Tableau VII) : ciel dégagé lors des sorties en premier quartier et ciel couvert lors des sorties en dernier quartier. Les civelles sont d'autant plus au fond que la nébulosité est faible. Cela renforce l'idée de l'influence de la lumière sur la répartition verticale des civelles dans la veine d'eau. GIBSON (1978) évoque la couverture nuageuse comme un facteur pouvant contrôler les mouvements migratoires de l'anguille en modulant l'intensité de la lumière lunaire.

La turbidité est un facteur qui est peu étudié si ce n'est à travers les fluctuations du débit fluvial (GASCUEL, 1987 ; MCGOVERN et MCCARTHY, 1992 ; DOMINGOS, 1992). II est certain que les variations du débit fluvial et de la turbidité sont fortement corrélées (Tableau II). Cependant, les débits modifient un grand nombre de facteurs environnementaux tels que : la vitesse du courant, les caractéristiques physico-chimiques de l'eau. En outre, sur l'Adour les relations débit et captures par unité d'effort ne sont pas clairement mises en évidence.

Nous confortons l'hypothèse de départ de l'influence de l'effet lumière sur les déplacements des civelles et sur le comportement lucifuge car les autres facteurs environnementaux identifiés comme des facteurs influant sur les déplacements verticaux de poissons ne peuvent intervenir dans le cadre de notre étude.

Dans la zone prospectée lors de l'échantillonnage, la colonne d'eau est homogène d'un point de vue de la température et de la salinité (DE CASAMAJOR, 1995). La stratification du milieu, avec la présence d'une halocline / thermocline, influence les déplacements verticaux des poissons dans la colonne d'eau (LEVY, 1990 ; APPENZELLER et LEGGETT, 1995). Ces travaux portent sur des espèces marines et montrent que les mouvements verticaux peuvent être bloqués dans une couche d'eau.

De même, la localisation des ressources trophiques vient moduler ces déplacements. Les civelles ne s'alimentent pas au cours de la traversée de l'estuaire (LEE et LEE, 1989) et la richesse trophique du milieu importe peu sur des individus qui ne s'alimentent pas. 


\section{PERSPECTIVES}

Le comportement lucifuge de la civelle résulte principalement de l'influence de 2 facteurs environnementaux. La turbidité de l'eau conditionne la pénétration de la lumière dans le milieu, suivant la nature et la quantité de matières en suspension. La phase lunaire, se traduit par une luminosité nocturne plus ou moins forte et vient moduler le facteur turbidité. Cet aspect du comportement est important car il conditionne l'accessibilité des individus aux engins de pêche utilisés sur l'Adour.

Ces résultats, sur la composante verticale de la migration des civelles, sont à compléter par une analyse de la composante horizontale de la migration. Les déplacements des civelles en zone maritime de l'estuaire de l'Adour résultent de la combinaison des variables hydrodynamiques : coefficient de marée et débit fluvial. L'interaction de ces 2 facteurs se traduit par une vitesse de courant résultante pendant le flot. Cette vitesse influence les densités de civelles présentes et leur localisation dans l'estuaire.

\section{BIBLIOGRAPHIE}

ANONYME, 1993. Embouchure de l'Adour: amélioration des accès au port de Bayonne, synthèse et actualisation des données existantes. CCl de Bayonne, DDE des Pyrénées-Atlantiques, $45 \mathrm{p}$.

APPENZELLER A.R., LEGGETT W.C., 1995. An evaluation of light-mediated vertical migration of fish based on hydroacoustic analyses of the diel vertical movements of rainbow smelt (Osmerus mordax). Can. J. Fish. Aquat., 52, 504-511.

BADIA J., PROUZET P., 1996. Modèles loglinéaires pour l'étude des associations entre captures par unité d'effort et variables hydroclimatiques. Aquat. Living Ressour., 9, 217-223.

BENZECRI J.P. et coll., 1979. L'analyse des données (Tome 2 : l'analyse des correspondances). DUNOD, Paris.

BRU N., DOSSOU-GBÉTÉ S., TRUONG-VAN B., 1996. Analyse exploratoire des captures de civelles d'anguille (Anguilla anguilla L.) dans l'estuaire de l'Adour. Lab. Math. Appli., UPPA, $32 \mathrm{p}$.

BRU N., DOSSOU-GBÉTÉ S., TRUONG-VAN B., 1997. Campagne d'échantillonnage 1996-1997 de la civelle d'anguille dans l'estuaire de l'Adour. Analyse exploratoire des données. Rapport Univ. Pau et Pays de l'Adour, 34 p.

CANTRELLE I., 1981. Etude de la migration et de la pêche des civelles (Anguilla anguilla L., 1758) dans l'estuaire de la Gironde. Thèse doc. 3ème cycle, Université de Paris VI, $238 \mathrm{p}$.

CASAMAJOR (de) M.N., 1995. Mesures de salinité et de température sur l'estuaire de l'Adour. Rapport interne, $37 \mathrm{p}$.

CASAMAJOR (de) M.N., 1998. Présentation de la campagne d'échantillonnage de la civelle d'Anguille (Anguilla anguilla L.) dans l'estuaire de l'Adour. Saison de migration 1996/1997. Rapp. Inst. Mil. Aquat., 34 p.

CASAMAJOR (de) M.N., BRU N., PROUZET P., 1996. La civelle d'Anguille (Anguilla anguilla L.) dans le bassin de l'Adour : productions et influence des conditions environnementales sur sa capturabilité. Rapport intermédiaire IFREMER, $41 \mathrm{p}$.

CHAMBERS J.M., HASTIE T.J., 1991. Statistical Models In Wadsworth S. \& Brooks/Cole Advanced Books \& Software, California, $608 \mathrm{p}$.

CHARLON N., 1980. Etude des populations de civelles de la région du bassin de l'Adour. INRA, Saint-Pée-sur-Nivelle (651330), 35 p. 
CHARLON N., BLANC J.M., 1982. Etude des civelles d'Anguilla anguilla L. dans la région du bassin de l'Adour. 1. Caractéristiques biométriques de longueur et de poids en fonction de la pigmentation. Arch. Hydrobiol., 93 (2), 238-255.

DOMINGOS I.M., 1992. Fluctuation of glass eel migration in the Mondego estuary (Portugal) in 1988 and 1989. Irish Fisheries Investigations Serie A (Freshwater), 36, 1-4.

FERNANDEZ J., VASQUEZ J., 1978. Las pesquerias de angulas en la provincia de holguin. Rev. Cub. de Invest. Pesq., 3(2), 48-61.

GANDOLFI G., PESARO M., TONGIORGI P., 1984. Environmental factors affecting the ascent of elvers, Anguilla anguilla (L.), into the Arno River. Oebalia, X (N.S.), 17-35.

GASCUEL D., 1987. La civelle d'Anguille dans l'estuaire de la Sèvre Niortaise. Biologie, Ecologie, Exploitation. Les publications du département d'halieutique, 4/1, $559 \mathrm{p}$.

GIBSON R.N., 1978. Lunar and tidal rhythms in fish. In Rhythmic Activity of Fishes, Thorpe J.E., London, New-York, San Francisco, 212 p.

JELLYMAN D.J., 1979. Upstream migration of glass-eels (Anguilla spp.) in the Waikato River. N. Z. J. Mar. Freshwater Res., 13(1), 13-22.

LEE T.W., LEE K.S., 1989. Daily growth increments and lunar pattern in otalith of the eel, Anguilla japonica, in the freshwater. Bull. Korean Fish. Soc., 22(1), 36-40.

LEVY D.A., 1990. Sensory mechanism and selective advantage for diel vertical migration in juvenile Sockeye Salmon, Oncorynchus nerka. Can. J. Fish. Aquat. Sci., 47, 1796-1802.

MCGOVERN P., MCCARTHY T.K., 1992. Elvers migration in the River Corrib system, western Ireland. Irish Fisheries Investigations Series A (Freshwater), 36, 25-32.

PROUZET P., MARTINET J.P., BADIA J., 1994. Caractérisation biologique et variation des captures de la grande alose (Alosa alosa) par unité d'effort sur le fleuve Adour (Pyrénées Atlantiques, France). Aquat. Living Resour., 7, 1-10.

TZENG W.N., 1985. Immigration timing and activity rhythms of the eel Anguilla japonica, elvers in the estuary of northern Taiwan, with emphasis on environmental influences. Bull. Jap. Soc. Fish. Oceanogr., 47/48, 11-28. 